\title{
Reversal reaction, nerve damage and steroid therapy in three multibacillary HIV positive patients
}

\author{
S. ARUNTHATHI, LAVANYA EBENEZER \& \\ C. KUMUDA \\ Branch of Medicine, Schieffelin Leprosy Research And Training \\ Center, Karigiri 632106
}

Accepted for publication 22 April 1998

The progress of three Indian HIV-positive patients with multibacillary leprosy has been recorded.

\section{Case 1}

A 38-year-old male gypsy was seen in 1992. On examination, he had multiple hypopigmented and erythematous lesions, some small, some large, on the trunk and limbs. The patches were hypoaesthetic, raised and warm, with well defined margins. He also had swelling of the feet and hands of 3 months duration.

The greater auricular, ulnar, ulnar cutaneous and radial cutaneous nerves were enlarged. The left ulnar nerve was tender. In the lower limbs, the lateral popliteal nerves were enlarged. There was extensive anaesthesia of the glove and stocking type. He had minimal clawing of the little finger of the left hand.

A diagnosis of borderline lepromatous leprosy in reversal reaction with severe ulnar neuritis was made.

\section{FAMILY HISTORY}

The patient was heterosexual, he had never used intravenous drugs or received a transfusion. He was married and had a $2 \frac{1}{2}$-year-old child. He had had sexual relations with another gypsy woman and subsequently developed a swelling in his groin and an ulcer on his penis, for which he had treatment.

Investigations revealed the following:

HB $11 \cdot 2 \mathrm{~g} \%$

WBC $10,000 \mathrm{~mm}^{3}$

Differential: neutrophils, 55; eosinophils, 16; lymphocytes, 20; monocytes, 8; basophils,

$1 ; \mathrm{ESR} ; 12 \mathrm{~mm} / \mathrm{h}$

Correspondence to: S. Arunthathi 
The liver function tests, blood sugar and blood urea were within the normal range. Chest $\mathrm{X}$-ray showed evidence of chronic bronchitis; sputum was negative for tubercle bacilli. The skin smears were positive for M. leprae B.I.1.6. Voluntary muscle testing (VMT) showed complete paralysis in the left ulnar nerve. VMT in the right ulnar nerve was 3 , and left ulnar 0 .

The lepromin (Mitsuda) reading was $7 \mathrm{~mm} \times 6 \mathrm{~mm}$, and the skin histopathology report (H\&E) revealed features typical of borderline tuberculoid leprosy with clumps of bacilli in reversal reaction, while the nerve biopsy was consistent with a diagnosis of borderline lepromatous leprosy with clumps of bacilli in reversal reaction.

Treatment was initiated with prednisolone $(60 \mathrm{mg} /$ day $)$ and Dapsone $(100 \mathrm{mg} / \mathrm{day})$.

\section{PROGRESS}

In hospital, the patient was found to have purulent urethral discharge and a penile ulcer. His VDRL test was reactive at 1 in 2 dilution and serum was positive by ELISA for HIV infection-1 and 2 , confirmed by Western blot. The venereal disease responded to a course of penicillin. His hospitalization 2 months after the start of therapy was complicated by herpes zoster of T7 and T8 dermatomes. He made an uneventful recovery from the zoster. Since the patient was ELISA positive for HIV infection, steroids were reduced more rapidly and the patient was started on clofazimine $100 \mathrm{mg}$ thrice daily as an anti-reaction drug for a period of 3 months.

The patient was started on the conventional WHO multibacillary-multidrug therapy (MBMDT) regimen. His voluntary muscle testing improved gradually, the left ulnar from 0 to 2 and the right ulnar from 3 to normal. While on MDT, he did not develop reversal reaction or neuritis. Since the disease was inactive in the skin lesions and nerves, and he was bacteriologically negative, he was released from treatment after 32 doses of the WHO regimen in March 1995. He has now completed 1 year of follow-up.

\section{Case 2}

A 25-year-old commercial sex worker who presented with multiple skin lesions of 2 years duration was seen in 1995.

On examination, she had multiple hypopigmented, well defined, erythematous, infiltrated, medium-sized plaques which were asymmetrical in distribution. The ulnar, radial cutaneous and ulnar cutaneous nerves were enlarged. The left posterior tibial and the left musculocutaneous nerves were also enlarged. She had stocking anaesthesia of the feet. She had minimal weakness of the orbicularis oculi muscle on the left side. A diagnosis of multibacillary leprosy BT/BB in severe reversal reaction was made.

\section{INVESTIGATIONS}

$\mathrm{Hb} 8.4 \mathrm{~g} \%$

WBC $13,000 / \mathrm{mm}^{3}$

Differential: neutrophils, 52; lymphocytes, 13; eosinophils, 30; monocytes, 5; ESR, $92 \mathrm{~mm} / \mathrm{h}$

The liver function tests and blood urea were within normal limits. VDRL was reactive at 1 in 256 dilution. She was positive by ELISA for 1 and 2 HIV infection. Chest X-ray showed 
prominent bronchovascular markings and enlarged right border of the heart. The skin smears were negative for M. leprae. Her VMTs in right and left ulnar nerves were 4 . The lepromin (Mitsuda) reading was $12 \mathrm{~mm} \times 12 \mathrm{~mm}$. The skin histopathology report suggested that the patient was probably upgrading from borderline lepromatous leprosy to borderline tuberculoid leprosy, while the nerve biopsy revealed a borderline tuberculoid picture with single small clumps of bacilli.

Treatment for the reversal reaction was initiated with prednisolone $(40 \mathrm{mg} / \mathrm{day})$ and clofazimine $100 \mathrm{mg}$ twice daily for a period of 3 months. She also received benzathine penicillin injection $2 \cdot 4$ megaunits.

\section{PROGRESS}

The patient was given haematinics for her anaemia and dapsone was stopped. When her haemoglobin had increased to $10.2 \mathrm{~g} \%$. Dapsone was restarted. The patient responded to treatment without any complications. She completed 24 pulses of MB-MDT and was released from treatment. She completed 1 year of follow-up.

\section{Case 3}

A 25-year-old male was seen in 1996. On examination, he had many hypopigmented, well defined and ill defined, dry, scaly, medium-sized anaesthetic skin lesions on the extremities. The skin lesions were warm and scaly. His ulnar, lateral popliteal, radial cutaneous, musculo-cutaneous, and posterior tibial nerves were bilaterally enlarged and non-tender. A diagnosis of borderline leprosy (BT-BB) in mild reversal reaction was made.

\section{FAMILY HISTORY}

This patient was a married heterosexual and had had sexual relations with a commercial sex worker 1 year previously. He developed a penile ulcer, for which he had received penicillin injections.

Investigations revealed the following:

$\mathrm{Hb} 11 \cdot 4 \mathrm{~g} \%$

WBC $9000 / \mathrm{mm}^{3}$

Differential; neutrophils, 54; eosinophils, 7; lymphocytes, 30; monocytes, 9; ESR, $95 \mathrm{~mm} / \mathrm{h}$

The liver function tests and blood urea were within normal range and blood VDRL was negative. Chest X-ray was normal. He was positive by ELISA for 1 and 2 HIV infection. His skin smears were negative for AFB. His VMT was normal. The lepromin (Mitsuda) reading was $15 \mathrm{~mm} \times 15 \mathrm{~mm}$, and the nerve histopathology report $(\mathrm{H} \& \mathrm{E})$ revealed a borderline lepromatous leprosy with epitheloid foci and bacilli singly and in small clumps within the nerve fascicles, while the skin biopsy findings were consistent with borderline tuberculoid leprosy.

\section{PROGRESS}

Treatment for the mild reversal reaction was initiated with chloroquine tablets, and he was started on the WHO MB-MDT regimen. 
The patient responded well to chloroquine. He completed five pulses of MB-MDT before being lost to follow-up.

\section{Discussion}

These three patients were 'high risk' for development of neuritis, as they had borderline leprosy and were in reversal reaction when admitted to hospital. A positive lepromin test was stated to be a risk factor for reversal reaction. ${ }^{1}$ HIV sera positivity has been associated with an increased incidence of neuritis and type-I lepra reaction, especially in MB leprosy patients. ${ }^{2}$ Histological characteristics of reactional skin lesions were also found to be similar in seropositive and seronegative leprosy patients. ${ }^{3}$

No differences have been reported in the response to steroid therapy among seropositive and seronegative leprosy patients with a lepra reaction. ${ }^{2}$ In our case study, the first male patient developed herpes zoster while on steroids, but he recovered from zoster without complications. He did not have delayed bacterial clearance of Mycobacterium leprae, or recurrent reversal reaction and neuritis. The second female patient also responded to corticosteroid treatment without complications. The third male patient did not receive corticosteroids, since he responded well to chloroquine.

HIV-infected patients with leprosy have been reported to respond well to anti-leprosy chemotherapy. ${ }^{4}$ The down-grading reactions towards lepromatous leprosy due to the combined immune deficiencies of HIV and leprosy ${ }^{5,6}$ did not occur in these three patients. Nor did the patients (cases 1 and 2) exhibit the poor outcomes in neuritis shown by the HIV-positive patients with leprosy in Zambia. $^{7}$

The selective tropism of HIV-1 for T-4 lymphocytes leads to T-cell depletion and the lowering of T-cell defences. The development of reversal reactions in these three patients is paradoxical. Presumably, these patients have immunological instability due to lowering of Tcell mediated immunity.

The case reports of all three cases raise several interesting points:

1. All three patients displayed clinical and histological evidence of reversal/upgrading reaction.

2. Though there is histological discordance between clinical and histological staging, all of them required the WHO MB-MDT regimen and responded promptly to conventional therapeutic treatment. The outcome was rewarding in terms of preventing permanent and progressive nerve damage. The first two patients responded well to corticosteroid therapy.

3. Concurrent HIV infection appears to result in anergy to lepromin, but in these three patients the lepromin test was strongly positive.

These patients will need to be followed up for years, since they may relapse, perhaps with unusual features.

\section{Acknowledgement}

We thank Mrs Glory Davidson for secretarial assistance. 


\section{References}

${ }^{1}$ Roche PW, Theuvenet WJ, Britton WJ. Risk factors for type-1 reactions in borderline leprosy patients. Lancet, 1991; 338: 654-656.

${ }^{2}$ Bwire R, Kawuma HJS. Type 1 reactions in leprosy neuritis and steroid therapy: the impact of the immunodeficiency virus. Transact $R$ Soc Hyg, 1994; 88: 315-316.

3 Sampaio EP, Caneshi JRT, Nery JAC, Duppre NC, Pereira GMB, Vieira LMM, Moreira AL, Kaplan G, Sarno EN. Cellular immune response to Mycobacterium leprae infection in human immunodeficiency virus-infected individuals. Infect Immun, 1995; 63: 1848-1854.

4 Blum L, Flageul B, Sow SL et al. Leprosy reversal reaction in HIV positive patients. Int J Lepr, 1993; 61: $214-217$.

${ }^{5}$ Patic AH. AIDS and leprosy (correspondence). Ind J Lepr, 1991; 59: 332-333.

6 Miller R. Leprosy and AIDS: a review of the literature and speculations on the impact of CD4 and lymphocyte depletion on immunity to Mycobacterium leprae. Int J Lepr, 1991; 59: 639-644.

7 Vreeburg AEM. Clinical observations on leprosy patients with HIV-1 infection in Zambia. Lepr Rev, 1992; 63: 134-140. 